https://doi.org/10.48009/1_iis_2010_29-34

\title{
THE PROCESS ARCHITECTURE OF INFORMATION SYSTEMS - HIGHER EDUCATION INSTITUTION'S MANAGERIAL TOOL
}

\author{
Srečko Natek, International School for Social and Business Studies, Slovenia, srecko.natek@mfdps.si \\ Dušan Lesjak, University of Primorska, Faculty of Management, Slovenia, dusan.lesjak@fm-kp.si
}

\begin{abstract}
Higher education institution's (HEI) management is dealing with a very complex system and its environment with a lot of demanding and related processes and activities dealing mainly with people. Various processes are found in HEI such as teaching, research, institutional, national and international cooperation, human resources, business, office, archival, decision support and intense data exchange processes. For effective and efficient HEI managed processes are essential, which cannot be achieved without careful planning and implementation of information system (IS) architecture. The process architecture of IS, focused on process coordination and integration, is the central part of the IS architecture. Because of the variety of HEI activities and special personnel requirements, human resources processes should be put at the centre of the architecture and tightly linked to other processes, especially academic and business processes, and upgraded with efficient decision support. The implementation of the process architecture demands global and contemporary computer support solutions, which will eliminate data and processes redundancy and ensure efficient and spatially-andtimely-unlimited access to HEI IS for all users, especially students, professors, researchers, staff, management and others. The process architecture of IS therefore serves as an important HEI managerial tool.
\end{abstract}

Keywords: Information System, Process Architecture of Information Systems, Management, Higher Education Institutions

\section{INTRODUCTION}

HEI management is a challenging, complex and highly dynamic task, since HEI are closely integrated in the higher education system at home and abroad, while at the same time they are also part of a wider local and regional environment. In order for management (administration) to successfully carry out its tasks, they have to ensure effective management of numerous inter-related processes, activities, events and especially people involved in them. For that they need a comprehensive review of business processes, focused on accuracy of content, complementarity, timelines and rational of their implementation. Processes must always be considered also in terms of information, because nowadays most of them are supported by information technology (IT). This is why the paper deals with the process architecture primarily in terms of information, i.e. as a whole of (inter-related and mutually dependent) business and information processes.

Avila [2] stresses the importance of adapting the IS architecture to an institution's strategies and business processes. Since strategies deal with an institution as a whole, the global process architecture of IS is the most important for their implementation. For that reason the paper focuses only on the global architectural aspect of processes. Research of detailed HEI processes requires different research approaches and is intended mainly for their organisation, implementation and the development of IT support. Regardless of the level of research, an organisation's processes are the backbone of its information development, which also applies to HEI. Therefore, the process architecture of IS is becoming an increasingly important HEI managerial tool.

Teaching and research are the most important HEI activities, but definitely not the only two. A diverse set of processes, activities and events at various levels take place within HEI. They relate to, intertwine with and complement each other, and are part of many processes in the regional and (usually very international) academic environment.

Every institution strives for well-organised and interrelated processes. If an institution has a sufficiently comprehensive, clear and documented overview of its processes, we say that it has the process architecture of IS. Whether it is useful for HEI management or not depends mostly on the content, long-term design and appropriateness of the process architecture of IS for effective management of HEI and its activities. Therefore, institutions try to plan the process architecture of IS as comprehensively as possible. 
Even though they often manage to achieve this, problems of discrepancy between the process architecture of IS and the actual implementation of processes arise. This is because HEIs change processes frequently and in most cases without checking whether they are consistent with the planned IS architecture. Moreover, they are often altered due to legal changes, organisational changes or changed or improved information support, even though HEIs know that changed processes are no longer consistent with the planned process architecture of IS. Coordination between the actual processes and the IS architecture is often left for the future when they will have more time, which is almost never the case.

It is even more difficult in institutions which do not plan and therefore do not have the process architecture of IS. This usually results in the duplication of business processes, information solutions, data sources and higher costs of implementing an institution's activities and therefore its decreased efficiency.

The above situation is especially common for HEI in which at least educational and research processes take place which are closely linked to processes in the environment, from the processes within HEI, cooperation with partner HEI and other organisations, also in an international environment, to the processes carried out by the ministry responsible for higher education at the national level. We must also not forget development, consultancy, publishing and seminar activities which often have the character of "business" and could be considered a separate activity, which is often closely linked to other HEI processes.

The implementation of all described activities and processes depends primarily on human resources available to HEI. Therefore, human resources management and development is a very important task of every management. This confirms the finding that human resources processes integrate all HEI activities and have a central role in the process architecture of IS. HEI human resources processes, such as planning the workload of staff carrying out educational and research work, are among the most complex HEI processes. According to Hislop [5] they are very important in the context of knowledge management as they represent the key to long-term success of HEI.

The absence of process architecture of IS usually means that processes of planning, scheduling and implementing educational and other processes are poorly inter-related, human resources processes are very time-consuming, it is difficult to plan and monitor the workload of staff and students, there are relatively many errors and hence also dissatisfaction of staff, students and administration. If poor integration with business processes, absence of decision-making support systems, particularly in the field of academic workload as highlighted by Zilli [11], poor documentation systems and extensive paper archives is added, risks and uncertainty of implementing HEI activities can become very high. If we add to these often non-regulated external security data archiving, the probability of facing various problems is even further increased.

Although the paper deals with the process architecture of IS, we do not want to diminish the significance of the data model which remains the most stable information model up to date since it changes very gradually, certainly more slowly than the process model. Its changing is primarily related to adapting to changed processes.

It is clear that the process architecture of HEI IS has to be carefully planned, implemented and used to control the development of HEI IS. It has become an important HEI managerial tool for managing changes of HEI processes, both in terms of content and the use of contemporary information technology. In this, it is reasonable to place HEI human resources processes in the centre of the process architecture of IS.

\section{PROCESS ARCHITECTURE OF HEI IS}

Ward [8] understands IS architecture as a global model of an institution's IS which in a very concise manner describes the main parts of IS and their mutual interdependence. Methodologically speaking, the description of IS architecture is a set of graphic models and textual descriptions, as demonstrated in Figure 1. Its first aspect is the process aspect (Global Process Model) which sets the process limits of an institution and places it in the environment. It is complemented with the data aspects (Global Data Model) whose special importance for the stability of IS is stressed by Adelman [1]. Whitten [10] notes that in environment of contemporary information technology security architecture (Global Security Model) is becoming increasingly important. It is a responsibility of a chief information security officer. White [9] deals with it on a strategic, tactical and operational level of the security model. IS architecture is completed with the technological aspect (Global IT Infrastructure Model) which is often neglected (Goncalves [4]), particularly in a 
contemporary internet environment that requires web applications development and multi-tier architecture. Lee [6] points out that content- and process-wise the most important is business logic as a middle tier. Gappa [3] states that HEI IS architecture should also be assessed from the point of view of students who are becoming increasingly demanding stakeholders and have to be taken into account.

In practice, IS architecture of an organisation is usually described as a combination of the above described models. The document of IS architecture may vary in scope, depending on the purpose, management requirements and willingness to invest financial and human resources in the preparation of IS architecture.

Although according to White [9] all the above models are important and interesting for every organisation, especially for HEI due to their diverse activities, we will hereafter limit our discussion to the process architecture of IS which we consider crucial for the overall quality of an organisation's IS architecture. We believe that this model should be planned first because processes are the core of every organisation's activities. Data is the means for successful implementation of processes, technology ensures efficient implementation of processes, and security architecture of IS reduces risks of carrying out processes, particularly in a sophisticated information technology environment.

In the introduction we found out that the research of process architecture should begin with human resources processes that integrate all HEI processes. This is why we placed human resources processes in the centre of the process architecture of IS, as demonstrated in Figure 2. Human resources processes in HEI are particularly exposed, since they are subject to cyclical dynamics of an academic year, and have to be previously formally and professionally accredited. Mills [7] notes that processes of accreditation of study programmes are the basis for future planning of education and implementation of study programmes. This is followed by multi-stage evaluation of student enrolment, intensive processes of detailed planning of human resources, calls for applications for new posts, interviews, selection of appropriate staff and making contracts for full or sessional employment. HEI human resources department and management often compare this process to "putting together a jigsaw". There are many possible combinations which depend mainly on the profiles of permanent staff and profiles of candidates who respond to our calls for occasional cooperation or partial fixed-term employment.
Similar can be said for research projects which often have a cyclical nature (e.g. various research projects). The difference is that in educational processes we are required to meet the needs of all staff (permanent employees and sessional professors) arising from study programmes opened for applications and actually carried out study programmes. In the case of research projects, we respond to calls individually and normally employ researchers, as this often is often required by our clients. In this context, research potential, profile and references of employed researchers are the key for the success of their application and later on for the implementation of research projects.

Employees' salaries should be calculated on the basis of their contractual obligations and work actually carried out. In Slovenia, this area is to a high extent regulated by law since our public higher education professors, researchers and other staffs are civil servants. However, it is also more or less specified in regulations which vary between HEI, both in details of classification of work and in time or value norms. Normally, the calculation system for employees and contracted personnel is different, not just regarding the structure of work, but also regarding the value. Therefore, it is no coincidence that in the calculation of salaries and contractual work payments many errors are made. Because the calculation system is so complicated and deferred to the entire academic year, employees and contracted personnel often do not know it well enough or have insufficient data on their salaries to verify the accuracy of the calculation.

In order to accurately calculate work carried out, inter-related processes must be taken into account. The basic processes are of course teaching activities (planned implementation of educational work, combining implementations and possible deviations in the actual implementation), complemented by the activities of human resources processes (actual contracts, annexes, staff changes, personal development, habilitations) and completed by business processes (calculation of salaries of employees and contracted personnel, material costs, finance, legal activities, administration, etc.).

It should be noted that in most HEI these processes are not properly integrated. Of course HEI processes are more or less inter-related, but the duplication of data, processes, computer solutions, additional manual calculations, many errors, etc. still occur, which results in tiresome coordination and often in 


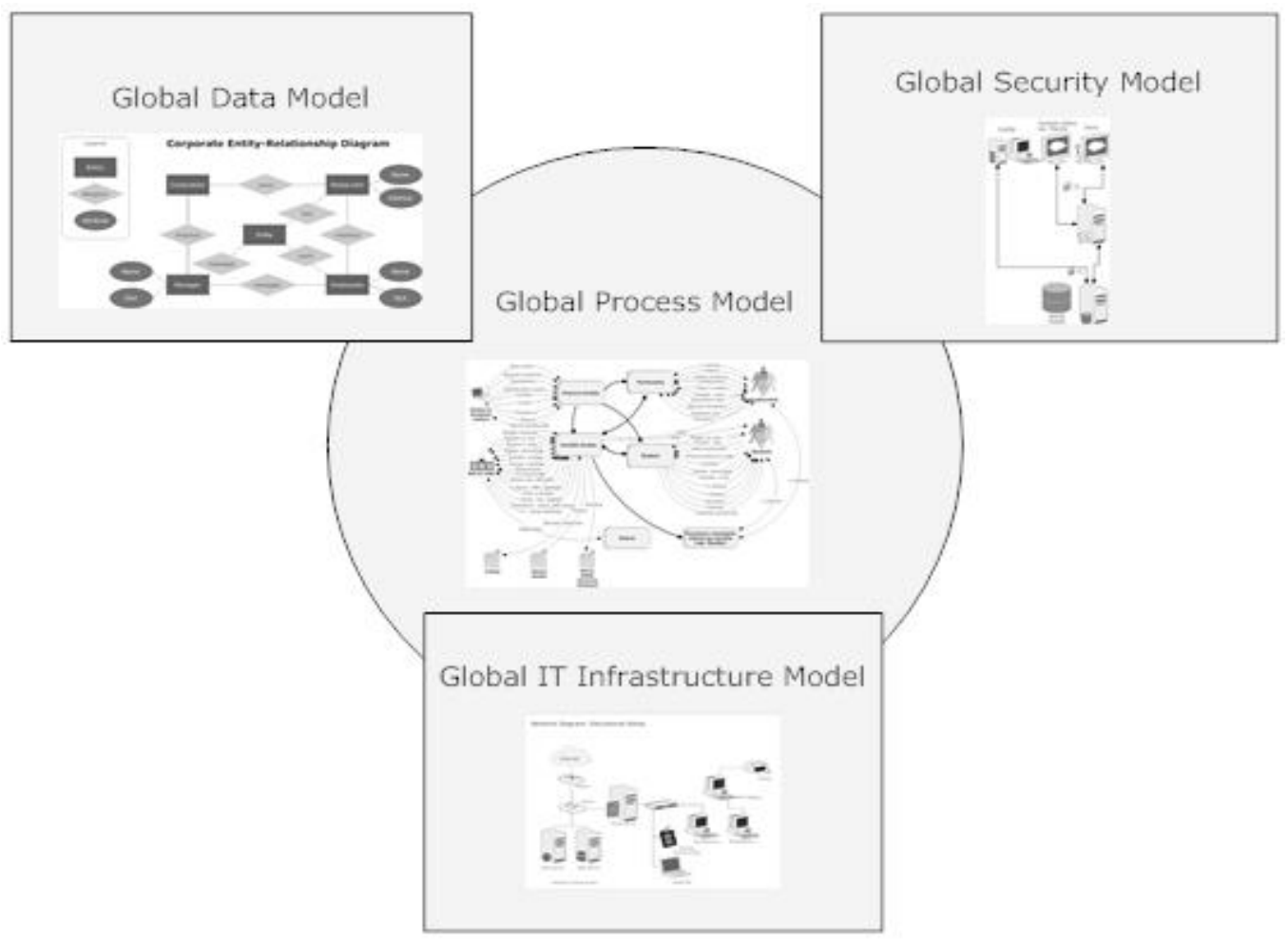

Figure 1. IS Architecture

disatisfaction of staff, management and, ultimately, students and their prospective employers.

The situation is similar in the case of research projects. They often increase the complexity of human resources processes, as sometimes research project funding is done through employees' salaries which means that the calculation of salaries includes educational and research work, which is part of various higher education processes.

Many HEI try to solve this architectural discrepancy between higher education, human resources and business processes with the help of data warehouses that combine all the key data on employees from data sources of mentioned processes. This is certainly a good solution for HEI that have yet to establish a comprehensive and fully integrated computer support for all their processes. Only then data warehouses and other systems supporting decision-making will serve its fundamental purpose, namely to support HEI management, and not to solve architectural discrepancies among key processes. According to Zilli [11], data warehouses are particularly useful for academic workload management.

The process architecture of IS has to be complemented with comprehensive office and archiving activities, normally subject of document systems, which is often a nightmare of HEI staff, not to mention high costs. The process architecture of IS must from the start ensure that all these processes are related to higher education, human resources and business processes, that documentation flow is highly automated and computer supported, and that the credibility of documentation is preserved. This of course requires an appropriate e-archive which gives the process architecture legally valid importance. This is not important only for formal/legal reasons, but it also provides a long-term guarantee of IS accuracy to all participants, both staff and students. By successfully upgrading these processes with external security data archiving, a long-term data security will be ensured and our investments in computer-based architecture of IS will be protected.

The process architecture of HEI IS must also be looked at in terms of its connections with the environment. Considering the content, business and 


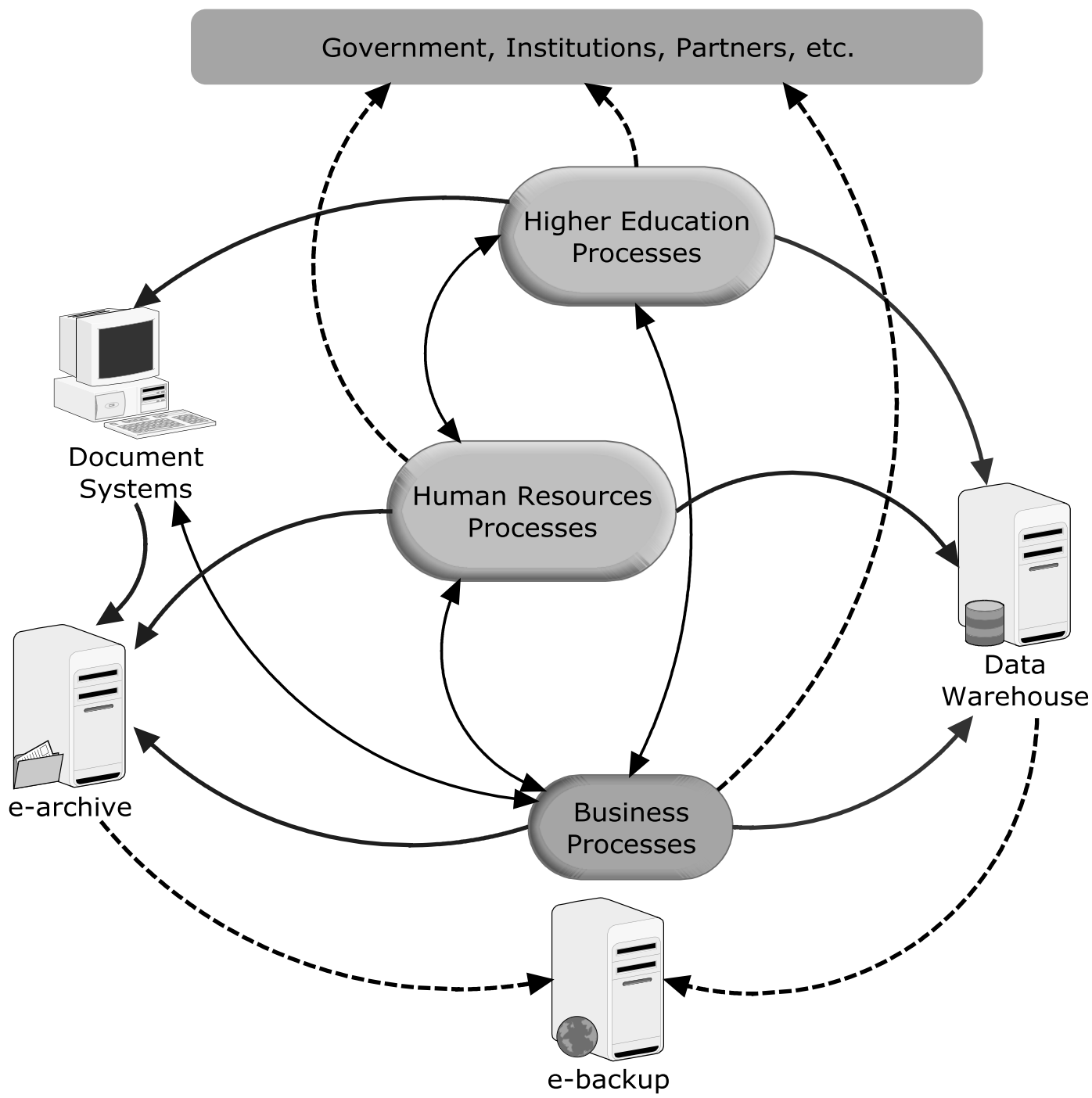

Figure 2. Process Architecture of HEI IS

legal aspects, it is necessary to mention data exchanges between HEI and the ministry responsible for higher education, the statistical office, the council and the national agency for higher education, the national research agency and other higher education and research institutions and business partners. It is often necessary to report data related to higher education, human resources and business processes. Reporting is easy in the case of appropriately computer-supported process architecture of IS. Otherwise, much effort has to be invested in the coordination of data.

It must be noted that the process architecture of IS in Figure 2 is highly simplified. It serves mainly as a starting-point and conceptual understanding of process integration of HEI and as a HEI managerial tool for managing IS development and also for managing and developing HEI themselves. In practice, described global processes must at the very least be devolved to the processes of the following levels, which could already be enough for the actual needs of projects of computer support for the whole process architecture of HEI IS.

\section{CONCLUSION}

Understanding of the process architecture of HEI IS and management decision to plan and implement it are necessary for the implementation of the process architecture of HEI IS, but unfortunately they do not necessarily mean its actual implementation. 
Planning of the process architecture of HEI IS is a challenge in itself, although in methodological terms it is not any different from planning any other organisation's architecture. However, the real professional and technological challenge is its actual implementation. This is only relevant as a comprehensive computer support using state of the art information technology.

Since a comprehensive solution which would with single technology support all the described processes does not exist, it is crucial that HEI choose a strategic provider of information support who will with their own solutions support the biggest part of their process architecture and in the future ensure the integration of computer support for the remaining processes in a way which will result in fully integrated processes.

When selecting a strategic provider HEI administration must follow the process architecture of IS which ensures organisational and contextual longterm objective of the HEI. They must also take into account providers' experience in developing and implementing complex computer solutions, their references in the areas of described processes, references in developing solutions using state of the

\section{REFERENCES}

1. Adelman, S. Moss, L., Abai, M. (2005). Data strategy, Addison Wesley, 9.

2. Avila, O., Goepp, V., Kiefer, F.. (2009). Understanding and Classifying Information System Alignment Approach, Journal of Computer Information Systems, IACIS, 50 (1), 2-14.

3. Gappa, J. M., Austin, A., Trice, A. G. (2007). Rethinking Faculty work: Higher Education's Strategic Imperative, John Wiley \& Sons, Inc., 11.

4. Goncalves, N. P., Danijel J. F. S. (2009): Information Systems Technological Infrastructure in the Polytechnic Institute of Setubal - Steps to Evolution. Available: http://eunis.dk/papers/p25.pdf

5. Hislop, D. (2009) Knowledge Management in Organizations, Oxford University Press, 2 ed., 239-256.

6. Lee, S. (2008). Collocation and Collation of Business Logic for Web Application art IT (e.g. multi-tiers web applications, especially for mass users such as students and professors). Offered information solutions must support all key processes such as human resources, higher education and business processes, and ensure management support by using data warehouses technology. Integration of various computer solutions must be implemented in a manner which will not result in data entry redundancy, large data transfers, etc. The described task of HEI administration can be better achieved if it is based on the process architecture of IS as the key HEI managerial tool for ensuring long-term success of IS development, resulting in the effectiveness and efficiency of the HEI itself.

The biggest challenge of HEI administration is to ensure the coherence between the process architecture of HEI IS and operative implementation of processes. If such coherence is not ensured, the process architecture of HEI IS becomes merely another information model which documents the objectives of the process architecture of HEI IS, but is no longer an effective HEI managerial tool.

Development, Journal of Computer Information Systems, IACIS, XLIX (1), 5766.

7. Mills, R. J., Hauser, K., Pratt, J. A. (2008). A Comprehensive two-level Framework for Information Systems Curriculum Design, Assessment and Improvement, Journal of Computer Information Systems, IACIS, XLVIII (4), 1-14.

8. Ward, J., Peppard J. (2002). Strategic Planning for Information Systems, 3 ed., Wiley, 192-204.

9. White, G. (2009). Strategic, Tactical \& Operational Management Security Model, Journal of Computer Information Systems, IACIS, XLIX (3), 71-75.

10. Whitten, D. (2008). The Chief Information Security Officer: An Analysis of the Skills Required for Success, Journal of Computer Information Systems, IACIS, XLVIII (3), 1519.

11. Zilli, D., Trunk Š., N. (2009). DSS for Academic Workload Management, Int. J. Management in Education, 3 (2), 185. 